\title{
Staff and Clinician Work-Life Perceptions after Implementing Systems-Based Improvements to Opioid Management
}

\author{
Brooke Ike, MPH, Laura-Mae Baldwin, MD, MPH, Sarab Sutton, BS, \\ Nicole Van Borkulo, MEd, Christine Packer, MEd, and \\ Michael L. Parchman, MD, MPH
}

Background: The Six Building Blocks Program is an evidence-based approach to primary care redesign for opioid management among patients with chronic pain. This analysis assesses the impact of implementing the Six Building Blocks on the work-life of primary care providers and staff.

Methods: Six rural and rural-serving primary care organizations with 20 clinic locations implemented the Six Building Blocks with support from a practice facilitator, clinical experts, and an informatics specialist. After 15 months of support, interviews and focus groups were conducted with staff and clinicians in each organization to stimulate reflection on the process and outcomes of implementing the Six Building Blocks Program. Transcripts of interviews and focus groups were coded and analyzed using template analysis. Once a set of themes was agreed on, the primary qualitative analyst revisited the source data to confirm that they accurately reflected the data.

Results: Overall, implementing the Six Building Blocks improved provider and staff work-life experience. Reported improvements to work-life included increased confidence and comfort in care provided to patients with long-term opioid therapy, increased collaboration among clinicians and staff, improved ability to respond to external administrative requests, improved relationships with patients using longterm opioid therapy, and an overall decrease in stress.

Conclusions: Clinicians and staff reported improvement in their work-life after implementing the Six Building Blocks Program to improve opioid medication management. Further research is needed on patient experiences specific to practice redesign programs. (J Am Board Fam Med 2019;32:715-723.)

Keywords: Chronic Pain, Family Physicians, Focus Groups, Opioids, Primary Health Care, Qualitative Research, Quality Improvement

Over half of all opioids in the US are prescribed by primary care clinicians. ${ }^{1}$ There is growing evidence that caring for patients with chronic pain on longterm opioid therapy (LtOT) contributes to stress

This article was externally peer reviewed.

Submitted 24 January 2019; revised 29 April 2019; accepted 2 May 2019.

From the Department of Family Medicine, University of Washington, Seattle, WA (BI, LMB, SS); Shift Results, Seattle, WA (NVB); Clearwater Valley Hospital and Clinics, Orofino, ID (CP); Kaiser Permanente Washington Health Research Institute, Seattle, WA (MP).

Funding: Grant R18HS023750 from the Agency for Healthcare Research and Quality (AHRQ). The contents of this product are solely the responsibility of the author and do not necessarily represent the official views of or imply endorsement by AHRQ or the U.S. Department of Health and Human Services. Additional support was provided by the National Center for Advancing Translational Sciences of the among clinicians and staff in primary-care settings. ${ }^{2}$ Clinicians, staff, and patients with chronic pain alike describe their interactions as challenging and frustrating. ${ }^{3-5}$ The clinician-patient relationship is strained by clinicians' negative perceptions

National Institutes of Health under Award Number UL1 TR002319. The content is solely the responsibility of the authors and does not necessarily represent the official views of the National Institutes of Health.

Conflict of interest: none declared.

Acknowledgements: We are grateful to the dedicated staff and providers at the participating WWAMI region Practice and Research Network clinics. Thank you for the care and persistence you brought to this important work.

Corresponding author: Brooke Ike, MPH, Department of Family Medicine, University of Washington, 4225 Roosevelt Way NE, Suite 308, Seattle, WA 98105-6099 (E-mail: bike2@uw.edu). 
of patients with chronic pain ${ }^{3,6-9}$ and ineffective clinician-patient communication. ${ }^{4,5,7,10-12}$ Clinicians struggle with uncertainty and discomfort regarding their ability to provide effective chronic pain management. ${ }^{13,14}$ At the same time, there is increasing pressure on primary care clinicians and staff to reduce opioid prescribing. This too can be stressful as these changes require difficult conversations with patients about tapering opioids.

Ironically, stresses and pressures related to caring for patients using LtOT are occurring simultaneously with an increasing emphasis on the role that clinician and staff well-being play in improving performance of health care systems. Bodenheimer and Sinsky ${ }^{15}$ introduced a fourth aim, improving the work-life of health care clinicians and staff, to the Institute for Health care Improvement's "Triple Aim" of enhancing patient experience, improving population health, and reducing costs. They suggest that ignoring the work-life of health care clinicians and staff interferes with achieving the "Triple Aim." 15 There is evidence that quality improvement programs can improve the work-life of clinicians and staff caring for patients using LtOT. ${ }^{16,17}$ Developing strategies that support clinician and staff wellbeing while promoting the highest quality care for patients using LtOT is critical.

The Six Building Blocks Program was developed to guide primary care practices in making systembased improvements in the management of patients using LtOT. ${ }^{18}$ The Six Building Blocks include 1) leadership support and consensus building; 2) revision and alignment of policies, patient agreements, and workflows; 3) tracking and monitoring patients; 4) planned, patient-centered visits; 5) identifying and connecting to resources for complex patients; and 6) measuring success. These concepts derived from approaches taken among 20 US practices identified as having exemplar, team-based clinical innovations. ${ }^{18}$

A study team from the University of Washington and the MacColl Center for Health Care Innovation at the Kaiser Permanente Washington Health Research Institute tested implementation of the Six Building Blocks through a facilitated program. ${ }^{19}$ This program included practice coaching, connection to resources, clinical education, and shared learning with other participating organizations and clinical advisors, described in more detail in Table 1. Following implementation, clinics saw a significant decrease in the number of patients using LtOT and the proportion of patients on high opioid doses. ${ }^{19}$. The Six Building Blocks Program includes many practical strategies suggested to improve work-life in the primary care setting, such as a focus on team-based care, standardizing workflows, and implementing team visit planning. ${ }^{17}$ In this study, we examine if the Six Building Blocks Program improved clinician and staff perceptions of the quality of their day-to-day work experience, referred to as "work-life."

\section{Methods \\ Study Setting}

We implemented the Team-Based Opioid Management Study within 6 rural-serving primary care organizations, totaling 20 clinic members of the WWAMI (Washington, Wyoming, Alaska, Montana, and Idaho) region Practice and Research Network, a primary care practice-based research network in the 5-state Washington, Wyoming, Alaska, Montana, and Idaho region. Five organizations were affiliated with Critical-Access Hospitals. The full-time equivalent of clinicians at the organizations ranged from 7.00 to 36.75 . The average number of patient visits per month ranged from 1270 to 9297 . Each organization formed an opioid improvement team, including a clinician champion and quality improvement lead, to implement the Six Building Blocks.

\section{Data Sources}

To assess clinician and staff perceptions of work-life after implementation, we analyzed end-of-study semistructured interviews and focus groups (Table 2). The interviews and focus groups were conducted by phone by a single study staff member (NV), and were audio recorded and transcribed. Each roughly 60minute session began with an oral informed consent process, approved by the University of Washington Human Subjects Division. We conducted interviews with members of the improvement team to ensure we heard their perspectives on implementation. To support participants' comfort in sharing openly, we conducted separate focus groups with staff and with clinicians, who self-selected based on interest and availability. Interview guides were developed to stimulate reflection on the process and outcomes of implementing the Six Building Blocks Program. Separate guides were created for the focus groups and interviews, but covered similar topics (Table 3). By 
Table 1. Six Building Blocks Program

\begin{tabular}{l}
\hline Building Block and Description \\
Leadership and consensus \\
Demonstrate leadership support and build organization- \\
wide consensus to prioritize more selective and \\
cautious opioid prescribing. \\
Policies, agreements, and workflows \\
Revise, align, and implement clinic policies, patient \\
agreements, and workflows for health care team \\
members to improve opioid prescribing and care of \\
patients with chronic pain. \\
Tracking and monitoring patient care \\
Implementing pro-active population management \\
before, during, and between clinic visits of all \\
patients on long-term opioid therapy. \\
Planned, patient-centered visits \\
Prepare and plan for the clinic visits of all patients on \\
long-term opioid therapy. Support patient-centered, \\
empathic communication for care of patients on long- \\
term opioid therapy. \\
Caring for complex patients \\
Develop policies and resources to ensure that patients \\
who develop opioid use disorder and/or who need \\
mental/behavioral health resources are identified and \\
provided with appropriate care, either in the care \\
setting or by outside referral. \\
Measuring success \\
Continuously monitor progress and improve with \\
experience. \\
Mechanisms of Support from the Six Building \\
Blocks Facititis
\end{tabular} Blocks Facilitation Team

Kickoff visit

Practice facilitation/coaching

Shared learning calls

Clinical education
Clinical champion and team continually provide visible leadership

Solicit and respond to feedback

Build organization-wide consensus

Revise and align policy and agreement in accordance to evidence, guidelines, and regulations

Redesign workflows to support policy

Develop tracking systems

Track patient care in order to pro-actively manage patients

Train on and implement workflows

Develop patient outreach and education

Train on patient-centered empathic communication

Identify assessment tools

Identify and connect to resources

Identify aims and success metrics

Measure success and continue improvements

\section{Description}

Site visit where all members of the organization's clinics (clinicians, nurses, medical assistants, front desk staff) come together to discuss making improvements to opioid management

Clinic-wide learning about evidence and guidelines

Small-group activity to self-assess current opioid management practices and to identify priorities for improvement

Guidance in creating an opioid quality improvement team

Ongoing guidance to develop and implement action plans to make improvements to opioid management based on best practices

Connection to tools and resources to support improvements (e.g., example policy, agreement, and workflows)

Monthly virtual learning collaboratives between participating sites

Brainstorm ideas for overcoming existing challenges

Share successful strategies and resources

Twice-monthly virtual clinical education run by a clinician pain specialist

Didactic presentations on topics identified as important by the sites (e.g., functional assessment, addiction assessment; exercise and pain)

Presentations and discussions of difficult cases the end of the data collection process the interviewer (NV) felt we reached data saturation as no new ideas were emerging.

\section{Data Analysis}

We used template analysis to examine how implementing the Six Building Blocks affected staff and clinician perceptions of their work-life. ${ }^{20} \mathrm{We}$ built our template (codebook) by identifying themes that emerged from any interview or focus group transcript text related to the questions of interest, "work-life changes" and "perceptions of patients." "Work-life changes" was coded when a participant discussed changes in day-to-day work experience 
Table 2. Qualitative Work-Life Study Data Sources

\begin{tabular}{|c|c|c|c|c|c|}
\hline Participant Group & $\begin{array}{l}\text { No. of } \\
\text { Sites }\end{array}$ & $\begin{array}{c}\text { No. of Participants } \\
\text { Per Site }\end{array}$ & Sample Method & $\begin{array}{l}\text { Type of Data } \\
\text { Collection }\end{array}$ & $\begin{array}{l}\text { Length, } \\
\text { Minutes }\end{array}$ \\
\hline Opioid improvement team & 6 & $\begin{array}{l}1 \text { to } 2 \text { (e.g., clinician } \\
\text { champion, quality } \\
\text { lead) }\end{array}$ & Purposive sampling & Interview & 60 to 90 \\
\hline Clinicians (MDs, DOs, PAs, NPs) & 5 & 5 to 10 & Convenience sample & Focus group & 45 to 60 \\
\hline Staff (MAs, nurses, front desk staff) & 6 & 5 to 10 & Convenience sample & Focus group & 45 to 60 \\
\hline
\end{tabular}

MD, medical doctors; DO, doctors of osteopathic medicine; PA, physician assistants; NP, nurse practitioners; MA, medical assistant.

related to Six Building Blocks implementation. "Perceptions of patients" was coded because we heard that interactions with patients using LtOT was a major component of the work-life experience and we looked for change in these perceptions. The core study team (BI, LMB, MP) read through the data within these 2 codes to identify emerging themes. These themes were developed into a set of primary and subcodes in an expanded template (codebook). The qualitative analyst then formally coded the text with the expanded template. The coding template was iteratively adjusted in response to the data until the coder (BI) felt that the codebook covered all work-life themes in the text (Table 4). The coded data were reviewed and organized by the study team (BI, LMB, MP, SS) to create a story of key changes in work-life perceptions, which was confirmed by rereading transcripts (BI, SS). All analyses were conducted in Dedoose 7.6.21 (Hermosa Beach, CA).

\section{Results}

Overall, clinicians and staff across the clinics indicated that participation in the Six Building Blocks Program improved their work-life experience. Reported improvements to work-life included increased confidence and comfort, increased collaboration and teamwork, improved ability to respond to external administrative requests (eg, from insurers, governmental organizations), and improved relationships with patients using LtOT. These improvements contributed to an overall reported decrease in stress among clinicians and staff.

\section{Increased Confidence and Comfort}

Clinicians and staff described an increase in confidence and comfort in 3 areas: the quality of care they provided, work processes, and their role in caring for patients using LtOT.

\section{Table 3. Questions from Quality Improvement Team Leader Interviews and Clinician and Staff Focus Groups}

\footnotetext{
Question

When you first signed up to take part in this study, what were you hoping would take place as a result of participating? To what degree did you meet these goals?

What are the major changes that your practice has made in the management of chronic opioid therapy patients?

Tell me about how the changes affect your daily work. What do you do differently now?

Reflecting on these changes that your practice has made in the management of chronic opioid therapy patients, what do you see as the key benefits (for the practice overall, for clinicians, for staff, for patients)?

Have there been any negative consequences of these changes that your practice has made in the management of chronic opioid therapy patients (for the practice overall, for clinicians, for staff, for patients)?

What surprised you as these changes in the practice or in your daily work started to be implemented? (FG)/What surprised you about the work? (I)

How have the changes that your practice has made influenced your attitudes toward your chronic opioid therapy patients? (FG)/How do you think attitudes toward chronic opioid therapy patients have changed through the implementation of this quality improvement initiative? (Among clinicians? Among staff? For you?)

A key objective of the project was to engage the clinical team in helping in the care of these chronic pain patients. What changes did your clinic make in the roles of the clinical team and how did this work out? What were the barriers to achieving this?
}

FG, focus group; I, interview. 
Table 4. Template of Data Coding Structure and Content

\begin{tabular}{ll}
\hline Primary Code & \multicolumn{1}{c}{ Sub-Code } \\
\hline Confidence and comfort & Quality of care \\
& Work processes \\
& Role \\
Collaboration & Between different roles \\
& Between the same role \\
Administrative & Insurance \\
& Liability \\
& Parent company \\
& Government \\
Improved relationships & Surprise at receptivity \\
& Fewer negative interactions \\
& Better relationship \\
Stress & Overall reduction in stress \\
\hline
\end{tabular}

\section{Increased Confidence and Comfort in Quality of Care}

After participating in the Six Building Blocks Program, staff and clinicians reported a higher level of confidence and comfort in the quality of the care they were providing to patients with chronic pain. As 1 clinician stated, "I feel confident managing my pain patients with or without opioids. This [the Six Building Blocks Program] gives you a confidence level that you are doing what is best for you and your patients." Clinicians and staff consistently reported that revising policies and agreements to reflect evidence-based guidelines and implementing consistent workflows to support these revisions seemed to significantly improve their comfort in caring for patients with chronic pain. As 1 clinician expressed, "a framework for managing these patients makes me feel a lot more comfortable about managing chronic pain patients than I ever did before." Some clinicians and staff provided examples of care improvement, such as "Focusing on concurrent prescribing of benzodiazepines and other things has really limited the risk of patient... harm." Feeling good about the quality and consistency of care also decreased clinician concerns about liability. "With the policy in place, I feel like there's a better focus on a method to manage the pain better and appropriately without feeling like there's a risk for liability."

\section{Increased Confidence and Comfort in Work Processes}

Clinicians and staff reported that standardized workflows developed through the Six Building
Blocks Program improved their care team's work, making it simpler. As 1 staff member said, "We have work systems in place where the monitoring is actually a little easier, and patients are getting used to those regular appointments every 3 months or whatever, that we can run through the pain follow up protocol and it just seems to be kind of getting more streamlined, not as cumbersome, not as dramatic."

\section{Increased Confidence and Comfort in Their Role}

Education on chronic pain and opioids, alongside implementation of consistent policies and teambased workflows, encouraged staff to function confidently as independent members of the team. One staff member said, "You do not feel like you have to go find a provider to say, 'Is it okay if I order a urine drug screen?' You know it is okay." Feeling confident and empowered to be active participants in the work came up repeatedly. "I know the [staff] feel much more comfortable saying this is what our doctors do, they are [the doctors] going to support my decision."

\section{Increased Collaboration and Teamwork}

Practice consistency also made it easier for clinicians and staff to function as a team and support one another in caring for patients using LtOT. As 1 clinician said, "Another advantage I see is covering for each other if someone's out on vacation or having a day off. There's less resistance in signing that prescription because you know that the standards are being followed uniformly." A staff member shared, "[Uniformity] has helped the staffthey just know, like he said, in a rural area we do struggle with staffing, so if someone calls in sick, then that MA can step in... everyone is on the same page with the process. So, it is made it easier."

Several clinicians and staff members reported a more collaborative working environment in which they were more able to communicate and brainstorm about the care of patients with chronic pain. As 1 clinician said, "The policy also helps us as providers communicate about the issue. When you do not have one [policy], you are kind of alone in dealing with it, so I think it is caused or led to more discussion between us about pain management." Increased trust among clinicians and staff throughout clinics was also reported as a key work-life improvement. One clinician enthusiastically shared, "As far as the 
culture change and everyone trusting each other better, there's no negatives in that part of it."

\section{Improved Ability to Respond to External Administrative Requests}

Another appreciated work-life improvement was the practical benefit of making it easier to respond to external administrative requests. Having established written policies, agreements, and workflows, along with tracking and monitoring systems, helped the practices with their interactions with parent companies, regulators, and insurance companies. "Insurance companies have been questioning us a lot more on our chronic opioid therapy patients and because we did [the Six Building Blocks Program], we have that information available at our fingertips to say here's our policy, here's what we've done, and we have it available, we have that pain contract when they ask for it, and when they do the case review. And that has been valuable."

\section{Improved Relationships with Patients}

At the study start, many clinicians and staff reported strained relationships with patients using LtOT. They expressed concerns over patient behaviors, such as early refill requests, angry outbursts, and possible illegal activity. Many did not think their patients would be willing to follow stricter policies or taper their opioid medication. Following the study, however, clinicians and staff reported several changes to these relationships with patients, including 1) a new recognition that patients are more receptive to changes in care approaches than originally presumed, 2) a reduction in negative emotional interactions, and 3) a positive shift in the kind of relationship clinicians and staff had with their patients using LtOT.

\section{New Recognition of Patient Receptivity to Change}

Participants reported being pleased with their patients' receptivity to changes in their opioid management, sometimes independently asking to taper. "The thing that surprised me was the number of patients that once they started churning through the standard care pathway, that said, 'Wow, I get it,' and then a lot of them just ended up tapering themselves ahead of us. I just was not quite prepared to see the patients engage." Clinicians and staff were not expecting the transition to new approaches to be well received by patients, and repeatedly voiced their surprise.
"I think the number of patients who were thankful to be tapered-that surprises me. Because usually when the pain patient gets to me, it is because they are mad that they did not get their prescription refilled quickly enough, so that surprises that patients did very much want to participate in being weaned off." (Staff member)

"Patients really aligned themselves with it and so many of them told me that, 'Gosh, I feel so much better. I still have pain, but it is no different.' And that surprised me." (Leadership)

"It has been well received, surprisingly. I expected a lot more pushback." (Leadership)

There were reports that while many patients responded well, receptivity varied. As 1 team lead explained, "Some of those patients that are so difficult that they refuse to sign a pain contract, they refuse to follow the rules and they stand firm, they do not budge. So we're able to sort of I guess weed those patients out. And other patients are like yes, I will follow the rules, I will do what I am supposed to, and I want to get off my narcotics."

\section{Reduction in Negative Emotional Interactions}

Participants often credited consistency and clear communication across the clinic with helping patients accept changes, thereby decreasing some of the negative emotional interactions that staff and clinicians shared at the start of the intervention. As 1 clinic manager described, "There's no more throwing fits out in the lobby and all the craziness that happens on the phone, you know, 'I want these meds and I want them now.' We've seen a decrease in the anxiety of the patients because they know what to expect, and everyone is holding to the same line, so I think it has helped all the way around." There were reported decreases in early refill calls, in challenging interactions with front desk staff, and in patients seeking to change clinicians. In addition, the clinician-patient relationship was reported to be less hostile. As 1 clinician described, "I used to have so many confrontational appointments where I'd have to have nursing staff standing outside the door because it was such an issue, being what someone called the evil doctor who would not prescribe narcotics and now everybody's on the same page and it is just an accepted philosophy of care with staff, providers, and with patients, it is accepted now." 


\section{Positive Shift in the Relationship with the Patient}

Not only was the clinician-patient dynamic reported to be less hostile, but in some clinics it was reported as more collaborative. "I can think of several other providers where it seems it is more of a team effort between the patient and the provider... there is more discussion [with patients] going on with those providers who have fully implemented and really gotten onboard with the program."

The clinical educational components of the Six Building Blocks Program were cited as a contributor to improved staff-patient relationships. Learning more about the complexities of caring for patients with chronic pain shifted perceptions, and many staff members reported seeing patients in a new way. "It is like, we got it. Ohh-the patient, they did not start out on cocaine, they did not start out on heroin. They started out on OxyContin and eventually got to the really dark place. So, it was an awareness, I think, that it is a progression. People do not start out to be addicts, it evolves into that. And that is what I learned from attending the webinars, from talking to people, from listening to the providers and their insight. So, it was a huge learning experience for me, and I hear the medical assistants and the LPNs say the same thing. It is like my gosh, these are people—these are people with problems, you know, and they are not the enemy. So, I think it has changed the way we look at that population."

Not all clinicians and staff changed their perceptions about patients using LtOT. One clinician shared, "I did not think too highly of them to start with, so it hasn't improved that... It reinforced my opinion that chronic pain management is a resource-intensive pain in the butt."

\section{Overall Stress}

Before implementation, many clinicians and staff reported that they felt stress related to their lack of clarity on how to provide quality care, practice variability, and challenges in their relationships with patients using LtOT, such as angry outbursts and early refill requests. Many reported that they felt like much of their clinical time was spent managing patients using LtOT. After implementation, clinicians and staff reported a decrease in stress. As 1 clinician succinctly put it, "emotional stress is $100 \%$ better." Because their clinics had consistent policies, clinicians and staff reported fewer negative emotional patient interactions and an increase in time for other kinds of patients. "I was surprised how much of the burden was lifted off of my shoulders in regard to managing pain patients. It did not feel like they were constantly asking anymore. They knew and understood what will happen, what will not happen with the policy, and it made it very almost mechanical to prescribe. It took a lot of the emotion out, which I think has been freeing, allowing us to focus on other sick patients that were not chronic pain patients." Organizational leadership perceived that there was less likelihood of staff and clinician turnover and the work day was more pleasant. However, despite consistent reports of less stress, clinicians and staff still mentioned that inadequate community resources to support patients using LtOT was a work-life stressor. As 1 clinician said, "I was jaded before, now there is a system in place... and it addresses liability issues. Less time talking about pain meds and more time focused on condition. However, there are not very many local resources for patients."

\section{Discussion}

Providers and staff reported improvements in their work-life after implementing the Six Building Blocks Program. Consistent with other studies of primary care clinic redesign, ${ }^{21,22}$ the structural changes put in place as a part of the Six Building Blocks Program improved both organizational and emotional aspects of the workplace. Increased confidence, comfort, collaboration, and teamwork improved clinician and staff perceptions of the practice environment and overall professional satisfaction. Clinic redesign has been shown to lead to high-functioning teams and improved professional satisfaction. ${ }^{22-24}$

The Six Building Blocks program's structural changes also led to improved relationships with patients using LtOT. This is consistent with findings that structural change related to opioid prescribing improves clinician-patient relationships. ${ }^{12}$ Staff and clinicians credited consistent policies, workflows, and expectations with a decrease in confrontational interactions with patients using LtOT, as well as an increase in perceived patient receptivity. Across all sites, staff and clinicians reported feeling surprised at the level of patient receptivity and engagement in their opioid management, sometimes independently asking to taper. Underestimating the patient's openness to change could 
stem from multiple, well-studied aspects of the clinician-patient relationship in the chronic pain setting: perception that patients using LtOT are drug seekers, ${ }^{4,8,10}$ lack of effective clinician-patient communication,, 11 and expectations of an antagonistic interaction. ${ }^{7,10,25}$ Previous studies have found increased empathy, decreased stigma, and shared decision making have reduced clinician-patient conflict and stress in the setting of chronic pain treatment and opioid prescribing. ${ }^{2,9,16,26-28}$ These findings suggest that it would be useful to augment the Six Building Blocks Program to include more focused training on empathy and stigma regarding patients using LtOT.

This study is limited in its reliance on selfreported outcomes of those clinicians and staff members who self selected to participate in interviews and focus groups. We did not actively recruit participants with disparate views about the program. In addition, we did not member check the findings with clinics. Although perceived improvements to work-life and decreased stress are clear, the data reflect clinician and staff perceptions, and may not represent "reality." Finally, our study is limited by not including the patient perspective.

\section{Conclusion}

Recent clinical guidelines by government agencies and professional organizations encourage more judicious use of opioids for chronic pain as a critical component of efforts to address the opioid crisis. ${ }^{29,30}$ At the same time, the call to action to address primary care clinician and staff burnout ${ }^{31}$ points to the importance of implementing opioid quality improvement programs that focus not only on patient safety, but also on the work-life conditions of staff and clinicians. The Six Building Blocks program has found that in addition to significant declines in both the proportion of patients on high-dose opioids and the total number of patients receiving opioids in participating clinics, ${ }^{19}$ implementing these system-based improvements to opioid medication management also improved clinician and staff perceptions of work-life quality. Future studies should assess the patient perspective specific to clinic redesign programs such as the Six Building Blocks.

To see this article online, please go to: http://jabfm.org/content/ 32/5/715.full.

\section{References}

1. Levy B, Paulozzi L, Mack KA, Jones CM. Trends in opioid analgesic-prescribing rates by specialty, U.S., 2007-2012. Am J Prev Med 2015;49:409-13.

2. Matthias MS, Parpart AL, Nyland KA, et al. The patient-provider relationship in chronic pain care: providers' perspectives. Pain Med 2010;11:1688-97.

3. De Ruddere L, Craig KD. Understanding stigma and chronic pain: a-state-of-the-art review. Pain 2016;157:1607-10.

4. Werner A, Malterud K. It is hard work behaving as a credible patient: Encounters between women with chronic pain and their doctors. Soc Sci Med 2003; 57:1409-19.

5. Kenny DT. Constructions of chronic pain in doctorpatient relationships: Bridging the communication chasm. Patient Educ Counsl 2004;52:297-305.

6. Phelan SM, van Ryn M, Wall M, Burgess D. Understanding primary care physicians' treatment of chronic low back pain: The role of physician and practice factors. Pain Med 2009;10:1270-9.

7. Henry SG, Bell RA, Fenton JJ, Kravitz RL. Communication about chronic pain and opioids in primary care: impact on patient and physician visit experience. Pain 2018;159:371-9.

8. Dobscha SK, Corson K, Flores JA, Tansill EC, Gerrity MS. Veterans Affairs primary care clinicians' attitudes toward chronic pain and correlates of opioid prescribing rates. Pain Med 2008;9:564-71.

9. Frantsve LM, Kerns RD. Patient-provider interactions in the management of chronic pain: current findings within the context of shared medical decision making. Pain Med 2007;8:25-35.

10. Crowley-Matoka M, Saha S, Dobscha SK, Burgess DJ. Problems of quality and equity in pain management: Exploring the role of biomedical culture. Pain Med 2009;10:1312-24.

11. Eggly S, Tzelepis A. Relational control in difficult physician-patient encounters: Negotiating treatment for pain. J Health Commun 2001;6:323-33.

12. Bieber C, Müller KG, Blumenstiel K, et al. Longterm effects of a shared decision-making intervention on physician-patient interaction and outcome in fibromyalgia: A qualitative and quantitative 1-year follow-up of a randomized controlled trial. Patient Educ Couns 2006;63:357-66.

13. Green CR, Wheeler JR, Marchant B, LaPorte F, Guerrero E. Analysis of the physician variable in pain management. Pain Med 2001;2:317-27.

14. Matthias MS, Krebs EE, Collins LA, Bergman AA, Coffing J, Bair MJ. "I'm not abusing or anything": patient-physician communication about opioid treatment in chronic pain. Patient Educ Couns 2013;93: 197-202.

15. Bodenheimer T, Sinsky C. From triple to quadruple aim: care of the patient requires care of the provider. Ann Fam Med 2014;12:573-6. 
16. Moral RR, Alamo MM, Jurado MA, de Torres LP. Effectiveness of a learner-centred training programme for primary care physicians in using a patient-centred consultation style. Family Practice 2001;18:60-3.

17. Wiedemer NL, Harden PS, Arndt IO, Gallagher RM. The opioid renewal clinic: a primary care, managed approach to opioid therapy in chronic pain patients at risk for substance abuse. Pain Med 2007; 8:573-84.

18. Parchman ML, Von Korff M, Baldwin LM, et al. Primary care clinic re-design for prescription opioid management. J Am Board Fam Med 2017;30:44-51.

19. Parchman ML, Penfold RB, Ike B, et al. Team-based clinic redesign of opioid medication management in primary care: impact on opioid prescribing. Ann Fam Med 2019;17:319-325.

20. King N. Using templates in thematic analysis of text. In: Cassell C, Symon G, eds. Essential guide to qualitative methods in organizational research. London, UK: Sage; 2004, 256-70.

21. Reid RJ, Coleman K, Johnson EA, et al. The group health medical home at year two: cost savings, higher patient satisfaction, and less burnout for providers. Health Aff (Millwood) 2010;29:835-43.

22. Sinsky CA, Willard-Grace R, Schutzbank AM, Sinsky TA, Margolius D, Bodenheimer T. In search of joy in practice: a report of 23 high-functioning primary care practices. Ann Fam Med 2013;11:272-8.

23. Gallagher RM. Empathy: A timeless skill for the pain medicine toolbox. Pain Med 2006;7:213-4.
24. Dobkin PL, De Civita M, Abrahamowicz M, et al. Patient-physician discordance in fibromyalgia. J Rheumatol 2003;30:1326-34.

25. Lyon C, English AF, Chabot Smith P. A team-based care model that improves job satisfaction. Fam Pract Manag 2018;25:6-11.

26. Mitchell P, Wynia M, Golden R, et al. Core principles and values of effective team-based health care. Washington, DC: Institute of Medicine; October 2012.

27. Burgess DJ, van Ryn M, Crowley-Matoka M, Malat J. Understanding the provider contribution to race/ ethnicity disparities in pain treatment: Insights from dual process models of stereotyping. Pain Med 2006; 7:119-34.

28. Banja JD. Toward a more empathic relationship in pain medicine. Pain Med 2008;9:1125-9.

29. Dowell D, Haegerich TM, Chou R. CDC guideline for prescribing opioids for chronic pain-United States, 2016. MMWR Recomm Rep 2016;65:1-49.

30. Washington State Agency Medical Directors' Group. Interagency Guideline on Prescribing Opioids for Pain, 2015. Agency Medical Directors' Group. http://agencymeddirectors.wa.gov/Files/ 2015AMDGOpioidGuideline.pdf. Accessed April 29, 2019.

31. Shanafelt TD, Dyrbye LN, West CP. Addressing physician burnout. The way forward. JAMA 2017; 317:901-2. 